\title{
High Sensitivity EFTEM Elemental Mapping with Unlimited Acquisition Time
}

\author{
Shixin Wang \\ Micron Technology, Inc. 8000 S. Federal Way, Boise, ID 83707
}

One of the applications of energy filtered TEM (EFTEM) using an EELS signal is to obtain elemental maps. An often encountered difficulty with EFTEM mapping is the signal being weak, either due to a small elemental concentration and/or signals from a large energy loss. The weakness of signals puts a significant limit on the sensitivity of EFTEM images.

The detectability of a signal depends on the signal-to-noise ratio (SNR). Statistically, SNR maybe expressed as [1]:

$$
\mathrm{SNR}=I_{0} /\left(I_{0}+h I_{b}\right)^{1 / 2}
$$

where $\mathrm{I}_{0}$ is the signal intensity, $I_{b}$ is the background intensity, and $h=\left[1+\operatorname{var}\left(I_{b}\right) / I_{b}\right]$. One approach to increase the detectability is to prolong the acquisition time. This can be illustrated by comparing the change of SNR with increased collection time. If we increase intensity, $I_{0}, N$ times to $I_{n}\left(I_{n}=N \cdot I_{0}\right)$, we have:

$$
\operatorname{SNR}\left(I_{n}\right) / \operatorname{SNR}\left(I_{0}\right)=(N)^{1 / 2} \cdot\left\{\left[1+h \cdot I_{b} / I_{0}\right] /\left[1+h \cdot I_{b} /\left(I_{0} \cdot N\right)\right]\right\}^{1 / 2}
$$

For a small elemental concentration, the right-hand side of the Eq. (2) approximates $N$. For large $N$, it is proportional to $N^{1 / 2}$. This nonlinear increase of SNR with increasing collection time is plotted in Fig. 1 (assuming $h=4$ ). The model described in Eq. (2) predicts unlimited improvement detectability with increasing acquisition time.

In practice, acquisition time is limited by sample drift. To overcome this limit, driftcompensation obtained from image cross-correlation may be applied. In this paper, a procedure for a drift-compensated, continuous EFTEM collection is described and demonstrated. The key points of this procedure include the following. (1) Images are acquired and saved iteratively. (2) A zero-loss image is acquired at each acquisition loop, along with other energy-loss images. (3) A cross-correlation calculation is performed on zero-loss image stack, and the resulting alignment data is applied to other images. (4) The aligned images of each energy window are added together, forming an image with intensity multiplied by the number of iteration, $N$.

A flowchart of a typical process is shown in Fig. 2. Sample drift within each acquisition loop is ignored. This assumption can be satisfied by allowing a certain sample settling time and by shortening individual exposure time. Based on the software configuration, the cross-correlation and summation can be performed either in-situ or ex-situ. Zero-loss images are used for crosscorrelation due to their high intensity. If the energy-loss images have high enough intensity, they may be used in cross-correlation calculation, and the zero-loss images can be omitted. Our test shows a much superior cross-correlation quality using zero-loss images than using the energyloss images. The iteration number $N$ has the same meaning as in Eq. (2). Essentially, $N$ (and so the total acquisition time) is unlimited. If necessary, the process may be divided into segments to allow manual alignment of the sample, electron beam, and spectrometer.

In our test, we used a DigitalMicrograph ${ }^{\mathrm{TM}}$ script to loop through each collection and saving step. The cross-correlation calculation was performed after collection using a Unix shell script calling programs in the IMOD package [2]. An example result is shown in Fig. 3. The location pointed by the arrow has a small oxide pocket, which only shows up after a large number of iterations ( $N$ $=130$ iterations, equivalent to 8.7 minutes exposure time).

\section{References}

[1] R. F. Egerton, Electron Energy-Loss Spectroscopy in the Electron Microscope, 2nd edition 
(Springer, 1996), p.277.

[2] IMOD home page: http://bio3d.colorado.edu/imod/.
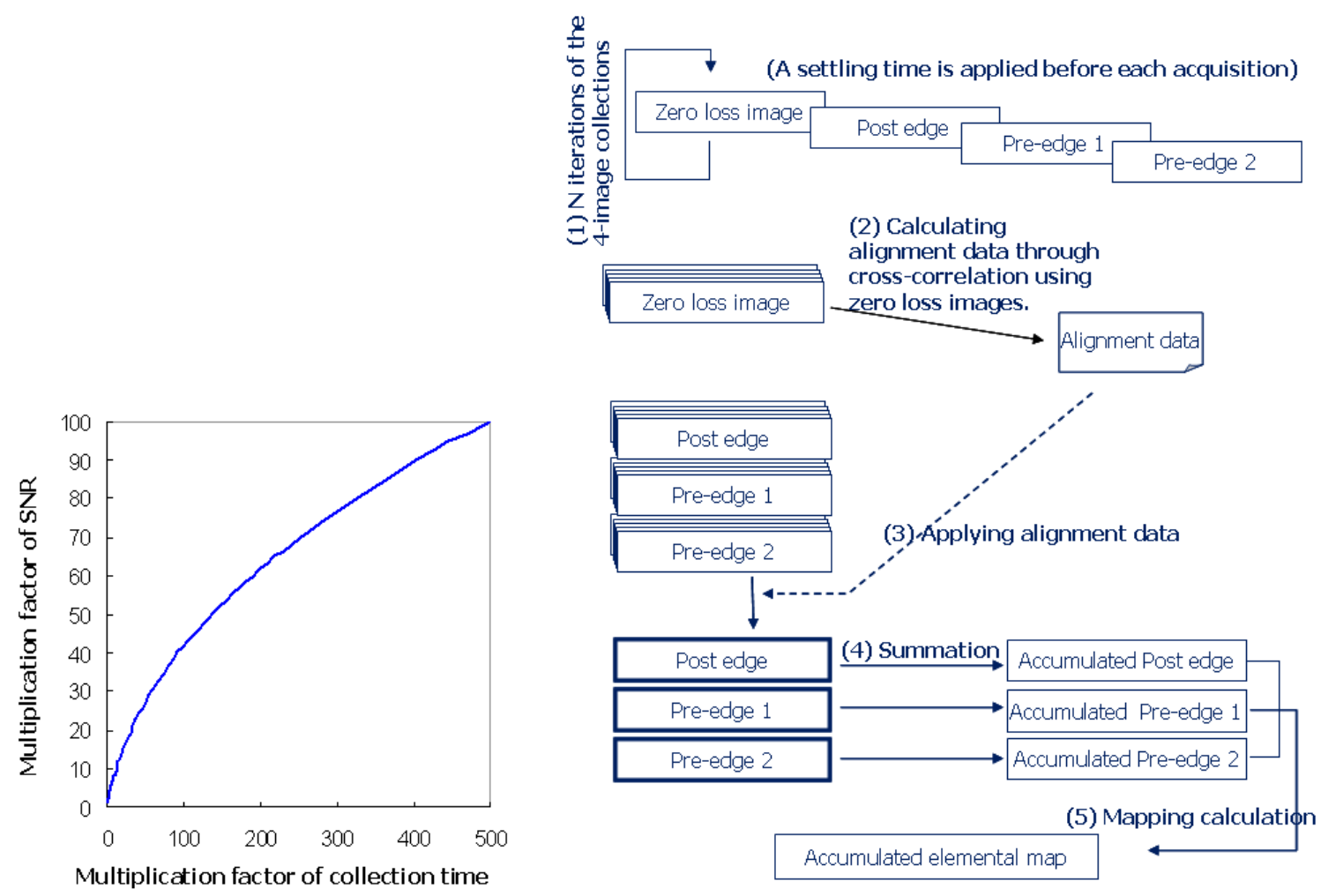

Fig. 1. The effect of increasing collection time on increasing SNR.

Fig. 2. Flowchart of the image collection and processing.
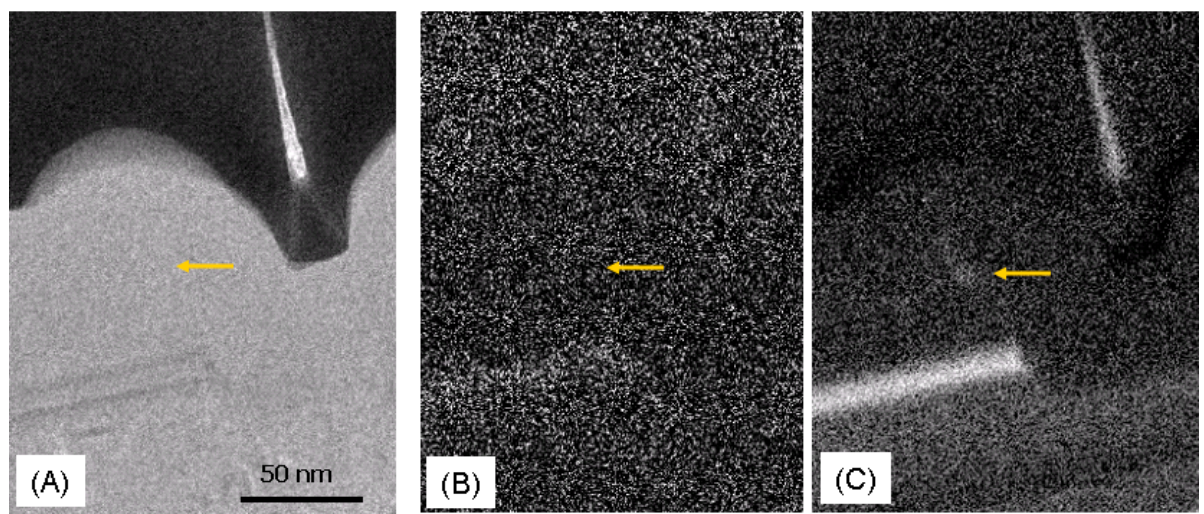

Fig. 3. (A) Zero-loss image. (B) O map with 2-second exposure. (C) Accumulated O map after 130 iterations with individual exposure time of 4 seconds. 